\title{
The Psychometric Hepatic Encephalopathy Syndrome score does not correlate with blood ammonia, endotoxins or markers of inflammation in patients with cirrhosis
}

\author{
Nina Kimer ${ }^{1,2}$, Lise Lotte Gluud ${ }^{1}$, Julie Steen Pedersen ${ }^{1}$, Juliette Tavenier ${ }^{3}$, Søren Møller ${ }^{2}$, \\ Flemming Bendtsen ${ }^{1}$ \\ ${ }^{1}$ Gastrounit, Medical Division, ${ }^{2}$ Centre of Diagnostic Imaging and Research, Department of Clinical Physiology and Nuclear Medicine, ${ }^{3}$ Clinical \\ Research Centre, University Hospital Hvidovre, Hvidovre, Denmark \\ Contributions: (I) Conception and design: N Kimer, F Bendtsen, S Møller; (II) Administrative support: N Kimer; (III) Provision of study materials or \\ patients: N Kimer, JS Pedersen, S Møller; (IV) Collection and assembly of data: N Kimer, JS Pedersen, S Møller, J Tavenier; (V) Data analysis and \\ interpretation: J Tavenier, N Kimer, LL Gluud; (VI) Manuscript writing: All authors; (VII) Final approval of manuscript: All authors. \\ Correspondence to: Nina Kimer. Gastro Unit, Medical Division, University Hospital Hvidovre, Kettegaard Alle 30, 2650 Hvidovre, Denmark. \\ Email: nina.kimer@regionh.dk.
}

Background: The pathogenesis of hepatic encephalopathy (HE) remains unclear but impaired clearance of gut-derived neurotoxins and increased systemic inflammation are thought to play key roles. The diagnosis is based on detection of neurophysiological and neuropsychometric abnormalities. The Psychometric Hepatic Encephalopathy Score (PHES) have been found to correlate with markers of systematic inflammation including interleukin 6, C-reactive protein (CRP) and tumor necrosis factor- $\alpha$ (TNF- $\alpha$ ). This study explores the associations between the PHES score and systemic inflammation, endotoxins and disease severity using baseline data from a trial involving patients with cirrhosis and minimal or no HE (NCT01769040).

Methods: Arterial blood was obtained during hepatic vein catheterization, from 54 patients [median age 55 (range, 33-70) years; $83 \%$ men] with decompensated but stable cirrhosis. None had clinical evidence of HE but 34 (55.6\%) had an abnormal PHES score indicating the presence of minimal HE. Relationships were sought between the PHES score and markers of systemic inflammation, high sensitivity-CRP, cytokines (SDF-1 $\alpha$, TGF-b1, IP-10, IL-6, 10 and 18, and TNF- $\alpha$; lipopolysaccharide (LPS), the lipopolysaccharide binding protein (LBP) and soluble CD14 (sCD14); and the blood ammonia.

Results: No significant relationships were found between the PHES score and any of the variables tested with the single exception of the correlation with serum IL-6 $(r=-0.29,95 \%$ confidence interval, -0.53 to -0.02, $\mathrm{P}=0.031$ ). No independent predictors of the PHES score were identified in regression analyses.

Conclusions: No predictive associations were identified between the PHES scores and circulating blood ammonia, endotoxins, or markers of systemic inflammation in this patient population.

Keywords: Cirrhosis; hepatic encephalopathy (HE); lipopolysaccharide binding protein (LBP); psychometry; portal hypertension; systemic inflammation

Received: 11 October 2019; Accepted: 10 February 2020; Published: 05 January 2021.

doi: $10.21037 / \operatorname{tgh} .2020 .02 .14$

View this article at: http://dx.doi.org/10.21037/tgh.2020.02.14

\section{Introduction}

Hepatic encephalopathy (HE) is a common complication of cirrhosis; its presence significantly affects outcome. Patients with cirrhosis and HE have a one-year mortality of more than $60 \%$ (1). The severity of HE varies from only a few psychometric abnormalities to severe cognitive dysfunction and coma. Minimal hepatic encephalopathy (MHE) is a neuropsychiatric condition in patients with cirrhosis who 
have no clinical features of HE but still have an impaired response to psychometric testing (2). People with cirrhosis and MHE have impaired sleep quality and a decreased quality of life $(3,4)$.

The presence of MHE can be detected using a range of neuro-psychometric and neurophysiological tools. The latter has been thoroughly testes and validated in numerous countries (5-8). Several precipitating factors of HE have been identified, in particular infection and dehydration (9). The pathogenesis of HE remains unclear, but impaired clearance of gut-derived neurotoxins such as ammonia may both facilitate and prolong episodes (10). Activation of systemic inflammatory responses may also play a role in enhancing the effects of ammonia and may also induce neuroinflammation, facilitating the development of $\mathrm{HE}$ in cirrhosis $(11,12)$. Several markers of inflammation have been demonstrated to be elevated in patients with MHE (13).

The degree of neuropsychiatric abnormality in HE varies considerably between different tests and between timepoints of assessment, even with comparable levels of impairment. Lipo-polysaccharides, interleukin-6 (IL-6), high-sensitive C-reactive protein (hs-CRP) and tumor necrosis factor- $\alpha(\mathrm{TNF}-\alpha)$ have in some studies shown to be independent predictors of an abnormal Psychometric Hepatic Encephalopathy Score (PHES) (14), and to correlate with circulating ammonia levels, the PHES score or even health related quality of life (15-17). However, studies are characterized by small cohort sizes and are mostly unconfirmed.

The aim of this study was to explore the associations between presence of MHE defined by the absence of clinical features of HE and an abnormal PHES score and arterial blood ammonia concentrations, markers of systemic inflammation and endotoxemia and standard measures of disease severity.

\section{Methods}

This study used baseline data from a randomized clinical trial undertaken in patients with decompensated cirrhosis but without clinical evidence of HE (NCT01769040) enrolled between February 2013 and December 2015 at Amager-Hvidovre University Hospital (18). The randomized clinical trial was approved by the Danish Medicines Agency (EudraCT No. 2012-002890-71) and by the Scientific Ethics Committee of the Capital Region of Denmark (journal No. H-2012-078). All participants gave informed written consent to participation in the trial. The present cross-sectional study involved 54 participants [median age 55 (range, 33-70) years; $83 \%$ men; 79\% alcohol-related cirrhosis, mean MELD score 11 (range, 6-25)]. Inclusion criteria were diagnosis of cirrhosis verified by clinical, biochemical, and ultrasound findings; clinical or ultrasound-verified ascites within the last three months; age 18 to 80 years; and portal hypertension with a hepatic venous pressure gradient $\geq 10 \mathrm{mmHg}$. Exclusion criteria were cardiac or respiratory failure, invasive cancer within the past five years, clinical or biochemical signs of infection, antibiotic treatment 14 days prior to inclusion, overt HE, kidney failure with serum creatinine above $200 \mu \mathrm{mol} / \mathrm{L}$, transfusion-requiring bleeding within one week prior to inclusion, blood hemoglobin level below $5.5 \mathrm{mmol} / \mathrm{L}$, continuous abuse of alcohol with symptoms of withdrawal; or expected survival of less than 3 months, as previously described $(18,19)$.

Ten participants had a history of one or more episodes of overt HE. All were clinically stable at the time of inclusion; none was actively misusing alcohol nor had done so for a minimum of 3 months; none took illicit drugs nor were prescribed psychoactive medication; 11 were receiving treatment with lactulose $15-60 \mathrm{~mL}$ daily, of which 9 had had previous episodes of overt HE. Two were prescribed lactulose $15-30 \mathrm{~mL}$ daily due to constipation.

Mental status was examined at baseline, using West Haven criteria (5); psychometric performance was assessed using the PHES test (8) using a cut off-of $<-4$ to define abnormality. Treatment of HE was instituted if indicated. An investigational program including systemic haemodynamic assessment was performed on the same day. Twenty-five $\mathrm{mL}$ of blood was drawn from the femoral artery during hepatic venous catheterization, immediately placed on ice, and samples of whole blood and EDTA plasma were stored at $-80{ }^{\circ} \mathrm{C}$ until analysed. All investigations were performed by trained physicians.

\section{Analyses of inflammation markers}

High sensitivity-CRP (hs-CRP), stromal cell-derived factor 1 alpha (SDF-1 $\alpha$ ), transforming growth factor beta 1 (TGF- $\beta 1$ ), interferon gamma induced protein 10 (IP-10), interleukins 10 and 18 (IL-10 and IL-18) were analysed with a commercially available enzyme-linked immunosorbent assay (ELISA) based on the sandwich principle (Quantikine, 
R\&D Systems Europe, Ltd. Abingdon OX14 3NB, UK). Sensitivity and the variation coefficient were reported previously (19).

The cytokines IL- 6 , TNF- $\alpha$, IL- $1 \beta$, IL-4, IL-10 and IL-18 were analysed in bulk with a high sensitive Luminex assay (Magnetic Luminex Performance, R\&D Systems Europe, Ltd., Abingdon OX14 3NB, UK). Less than 0.5\% cross-reactivity and interference between agents tested is seen. Sensitivity and variation coefficient have been reported previously $(19,20)$.

\section{Measurement of endotoxin markers}

Lipopolysaccharide (LPS), lipopolysaccharide binding protein (LBP) and the functional receptor for LPS, soluble CD14 (sCD14), were measured in EDTA plasma. LPS levels were determined using the Limulus Amoebocyte Lysate kinetic chromogenic methodology, optimized for sensitivity (Vaiomer SAS, Toulouse, France) with a commercially available kit (Charles River) $(21,22)$. The quantifiable limit for LPS was $0.024 \mathrm{EU} / \mathrm{mL}$. LBP and sCD14 levels were determined using a commercially available solid-phase ELISA based on the sandwich principle. The quantifiable limits for LBP and sCD14 were 3.5 and $0.13 \mu \mathrm{g} / \mathrm{mL}$, respectively.

\section{Statistical analysis}

Patient characteristics in the two groups divided by PHES score (below -4 and -4 or higher) were compared using the Mann Whitney U-test. Correlations between the numerical PHES scores and blood markers were performed using Spearman Rank test. Logistic regression analysis based on dichotomous division of the PHES score at the $<-4$ threshold were undertaken to determine predictors of the PHES score (as defined by German normative data, since no Danish normative data are available (8), and multivariate analysis with backwards elimination were performed to determine if any factor independently predicts an abnormal PHES score. Analyses were performed using Graph Pad Prism version 7.0 and SAS version 9.4. P-levels of 0.05 or less were considered significant. To avoid multicollinearity, albumin, coagulation factors and bilirubin levels were not included in the logistic regression.

\section{Results}

Data on effects and outcomes of the clinical trial is reported elsewhere $(18,19)$. The median PHES score at inclusion was -6 (-15 to 3$)$. Overall 34 patients (63\%), had an a PHES score below -4. Patient characteristics in the two groups (PHES score $\geq-4$ or $<-4$ ) are presented in Table 1. The ten patients who had a previous history of one or more episodes of overt HE were more likely to have an abnormal PHES score than those who had not (Table 1). Otherwise there were no significant differences in clinical, demographic or standard biochemical variables, between the two groups.

No significant correlations were observed between blood ammonia, markers of systemic inflammation and of bacterial translocation and the PHES score, with the single exception of the correlation with serum IL-6 [ $\mathrm{r}=-0.2937,95 \%$ confidence interval (CI), -0.5264 to $-0.0201, \mathrm{P}=0.0311$ ] (Table 2). Logistic regression analysis of single parameters did not reveal an association to abnormal PHES, Table 3.

Multivariate analysis with backward elimination of the inflammation markers IP-10, TGF- $1 \beta$, IL-6, SDF- $1 \alpha$, IL-18, IL-10, hs-CRP, IL- $1 \beta$ and TNF- $\alpha$, endotoxemia markers LBP and LPS, as well as markers for disease severity (Meld score, Child score, creatinine, glomerular filtration rate (estimated by chrome EDTA clearance), arterial ammonium, white blood cell counts, and sodium levels) left no parameters with significant values as prognostic indicators of an abnormal PHES score.

\section{Discussion}

In this cohort of patients with decompensated but stable cirrhosis approximately two-thirds had an abnormal PHES score. Patients with a history of previous episodes of HE were more likely to have an abnormal PHES score. We found a weak association between PHES score and IL-6, but no further evidence to suggest an association between other markers of inflammation, arterial ammonia or disease severity such as MELD and Child score to an abnormal PHES. Accordingly, this study did not find convincing evidence that PHES is associated with ammonia, inflammation or endotoxins. The small sample size and the relatively small number of patients could be important to the interpretation of the results and our findings should be assessed in larger studies.

The PHES score is a validated tool for diagnosing MHE and has been shown to predict development of overt HE in patients with cirrhosis (23). Associations between an abnormal PHES score and biological markers have also been examined, with the aim to find pathogenetic factors that facilitate $\mathrm{HE}$ and to find new targets for treatment of 
Table 1 Characteristics in the included trial participants with cirrhosis, by PHES score

\begin{tabular}{|c|c|c|c|}
\hline Characteristics & PHES $\geq-4(n=20)$ & PHES $<-4(n=34)$ & Significance $(P)$ \\
\hline Age (yr) & $56[34-74]$ & $55.5[33-68]$ & 0.9 \\
\hline Proportion of men & $16(80 \%)$ & $29(85 \%)$ & NA \\
\hline Previous hepatic encephalopathy & $1(5 \%)$ & $9(26 \%)$ & 0.02 \\
\hline PHES score & $-1.5(-4$ to 3$)$ & $-8(-15$ to -5$)$ & NA \\
\hline Child-Pugh score & $7.5[7-10]$ & $8[7-12]$ & 0.85 \\
\hline MELD score & $10.5[6-17]$ & $11[6-25]$ & 0.57 \\
\hline Plasma albumin (g/L) & $30.5[23-40]$ & 29.5 [21-43] & 0.5 \\
\hline Sodium (mmol/L) & $136[141-132]$ & $135[127-149]$ & 0.07 \\
\hline Bilirubin $(\mu \mathrm{mol} / \mathrm{L})$ & 18.5 [9-82] & $21[5-166]$ & 0.24 \\
\hline Arterial ammonia $(\mu \mathrm{mol} / \mathrm{L})$ & 46 [20-88] & 53.5 [19-94] & 0.31 \\
\hline WBC $\left(10^{9} / \mathrm{L}\right)$ & $6.0(3.5-10.8)$ & $6.7(2.6-16.9)$ & 0.70 \\
\hline TNF- $\alpha(p g / m L)$ & $8.6(2.4-22.5)$ & $8.9(3.6-35.8)$ & 0.36 \\
\hline IL1 $\beta(p g / m L)$ & $0.18(0.02-1.16)$ & $0.11(0.01-0.61)$ & 0.51 \\
\hline Hs-CRP (ng/mL) & $4,426[837-25,829]$ & $6,751[138-38,779]$ & 0.26 \\
\hline IL-10 (pg/mL) & $0.38(0.07-1.09)$ & $0.17(0.01-2.71)$ & 0.16 \\
\hline IL-18 (pg/mL) & $334.2(132.4-3,080.4)$ & $267.3(92.5-2,939.0)$ & 0.55 \\
\hline $\mathrm{SDF}-1 \alpha(\mathrm{pg} / \mathrm{mL})$ & $3,736[2,231-6,137]$ & $3,698[2,753-5,273]$ & 0.60 \\
\hline IL-6 (pg/mL) & $2.68(0.27-25.96)$ & $6.16(0.60-201.38)$ & 0.08 \\
\hline TGF-1 $\beta$ (pg/mL) & $10,249(4,241-55,776)$ & $7,627(2,598-37,529)$ & 0.08 \\
\hline $\mathrm{IP}-10(\mathrm{pg} / \mathrm{mL})$ & $249.8(140.8-407.3)$ & $231.3(81.4-1,166.3)$ & 0.45 \\
\hline LPS (EU/mL) & $0.09(0.02-0.20)$ & $0.09(0.02-0.18)$ & 0.49 \\
\hline LBP $(\mu \mathrm{g} / \mathrm{mL})$ & $6.7(3.4-16.1)$ & $7.2(3.4-24.8)$ & 0.38 \\
\hline
\end{tabular}

Data are expressed as median and range, unless otherwise stated. PHES, Portosystemic Hepatic Encephalopathy Score; MELD, Model for End Stage Liver Disease; WBC, white blood cells; LPS, lipo-polysaccharide; LBP, lipo-polysaccharide binding protein; EU, endotoxin unit; hs-CRP, high-sensitive C-reactive protein.

HE $(13,14,24,25)$. In a cohort of eighty patients with MHE relations between heart rate variability, PHES score and inflammation markers, IL-6 significantly correlated with heart rate variability and neuropsychiatric performance (24). In a prospective trial with 22 months of follow-up, PHES score correlated with both IL- 6, TNF- $\alpha$ and CRP; moreover, IL-6 was associated with an abnormal electro encephalogram (14). A small pilot study has even shown that inflammation markers may exacerbate the neurocognitive effects of ammonia in HE (26). However, in a large cohort, levels inflammation markers were higher in patients with MHE, but presence of MHE was independent of ammonia concentration and severity of cirrhosis. This raises the question of clinical relevance of inflammation markers and their validity as prognostic predictors. Older trials with small samples have indicated that TNF- $\alpha$ is involved in the pathogenesis of HE and MHE $(16,27,28)$. Recently a randomized trial on lactulose plus albumin versus lactulose alone for 120 people with overt $\mathrm{HE}$ found a greater decrease of TNF- $\alpha$, IL- 6 and IL-18 in the lactulose plus albumin treatment group (29). However, in another recent randomized trial including patients with cirrhosis and MHE or no cognitive impairment, no such effect was demonstrated (19). Further research into the pathogenesis 
Table 2 Relationship between the PHES score and markers of inflammation and disease severity in patients with cirrhosis

\begin{tabular}{|c|c|c|c|c|}
\hline Variable & Correlation coefficient $(r)$ & $95 \% \mathrm{Cl}$ & Significance $(P)$ & Pairs (n) \\
\hline WBC $\left(10^{9} / L\right)$ & -0.0243 & -0.30 to 0.25 & 0.86 & 54 \\
\hline TNF- $\alpha(p g / m L)$ & -0.02 & -0.29 to 0.26 & 0.91 & 54 \\
\hline $\mathrm{IL}-10(\mathrm{pg} / \mathrm{mL})$ & 0.21 & -0.12 to 0.51 & 0.2 & $38^{A}$ \\
\hline IL-6 (pg/mL) & -0.29 & -0.53 to -0.02 & 0.03 & 54 \\
\hline Hs-CRP (ng/mL) & -0.17 & -0.43 to 0.11 & 0.22 & 54 \\
\hline TGF- $\beta 1$ (pg/mL) & 0.19 & -0.09 to 0.44 & 0.17 & 54 \\
\hline $\mathrm{IP}-10(\mathrm{pg} / \mathrm{mL})$ & 0.03 & -0.25 to 0.30 & 0.83 & 54 \\
\hline SDF-1 $\alpha(p g / m L)$ & 0.01 & -0.26 to 0.29 & 0.92 & 54 \\
\hline IL-18 (pg/mL) & 0.05 & -0.23 to 0.32 & 0.73 & 54 \\
\hline IL-1 $\beta(p g / m L)$ & 0.05 & -0.23 to 0.32 & 0.74 & $53^{B}$ \\
\hline Ammonium ( $\mu \mathrm{mol} / \mathrm{L})$ & -0.16 & -0.43 to 0.13 & 0.26 & 51 \\
\hline MELD & -0.16 & -0.49 to 0.12 & 0.24 & 54 \\
\hline Child & -0.28 & -0.52 to -0.01 & 0.04 & 54 \\
\hline Albumin (g/L) & 0.1523 & -0.1283 to 0.4104 & 0.27 & 54 \\
\hline
\end{tabular}

A, IL-10 were below detection level in 16 patients. ${ }^{\text {B }}$, IL-1 $\beta$ were below detection level in 3 patients. PHES, Portosystemic Hepatic Encephalopathy Score; MELD, Model for End Stage Liver Disease; WBC, white blood cells; hs-CRP, high-sensitive C-reactive protein.

Table 3 Variables associated with abnormal PHES score (<-4) by logistic regression

\begin{tabular}{|c|c|c|c|c|}
\hline Variable & Hazard ratio & $95 \% \mathrm{Cl}$ & Significance $(P)$ & SE \\
\hline Child score & 0.74 & 0.47 to 1.17 & 0.19 & 0.173 \\
\hline MELD score & 0.99 & 0.87 to 1.13 & 0.89 & 0.065 \\
\hline Albumin (g/L) & 1.07 & 0.96 to 1.19 & 0.22 & 0.058 \\
\hline Ammonia $(\mu \mathrm{mol} / \mathrm{L})$ & 0.99 & 0.97 to 1.023 & 0.75 & 0.092 \\
\hline WBC $\left(10^{9} / L\right)$ & 1.02 & 0.86 to 1.22 & 0.83 & 0.092 \\
\hline TNF- $\alpha(p g / m L)$ & 1.05 & 0.97 to 1.15 & 0.21 & 0.044 \\
\hline IL-1 $\beta$ (pg/mL) & 6.71 & 0.58 to 77.27 & 0.13 & 8.366 \\
\hline Hs-CRP (ng/mL) & 1.00 & 0.99 to 1.00 & 0.98 & $<0.001$ \\
\hline IL-10 (pg/mL) & 1.40 & 0.57 to 3.42 & 0.46 & 0.639 \\
\hline IL-18 (pg/mL) & 1.00 & 0.99 to 1.001 & 0.27 & $<0.001$ \\
\hline SDF-1 $\alpha(p g / m L)$ & 1.00 & 0.99 to 1.001 & 0.23 & $<0.001$ \\
\hline IL-6 (pg/mL) & 1.00 & 0.99 to 1.02 & 0.93 & $<0.007$ \\
\hline TGF-1 $\beta$ (pg/mL) & 1.00 & 0.99 to 1.00 & 0.07 & $<0.001$ \\
\hline IP-10 (pg/mL) & 1.00 & 0.99 to 1.00 & 0.83 & 0.001 \\
\hline $\operatorname{LBP}(\mu \mathrm{g} / \mathrm{mL})$ & 1.00 & 0.89 to 1.13 & 0.98 & 0.061 \\
\hline LPS (EU/mL) & $5,362.249$ & 0.06 to $4.83 \times 10^{8}$ & 0.14 & $31,213.04$ \\
\hline
\end{tabular}

$\mathrm{Cl}$, confidence interval; SE, standard error; PHES, Portosystemic Hepatic Encephalopathy Score; MELD, Model for End Stage Liver Disease; WBC, white blood cells; LPS, lipo-polysaccharide; LBP, lipo-polysaccharide binding protein; EU, endotoxin unit; hs-CRP, high-sensitive C-reactive protein. 
of both MHE and HE and their interaction is warranted. Also, novel biological markers, specifically useful for diagnosing MHE and scaling severity of $\mathrm{HE}$, have not been identified. This is in agreement with the present study, which was unable to demonstrate an association between inflammatory cytokines to an abnormal PHES or overall disease severity.

In animal models, the anti-inflammatory drug ibuprofen has proven beneficial in MHE $(30,31)$. In humans, several treatment options for HE and MHE have been explored, with focus on reducing ammonia such as rifaximin, nonabsorbable disaccharides and branched chain amino acids (32-34), all drugs with a beneficial effect on preventing recurrent episodes $\mathrm{HE}$ and non-absorbable disaccharides being a corner-stone in the treatment of overt HE. Further trials with clinically relevant outcomes evaluating drugs for prevention and treatment of MHE are highly warranted. Other agents focusing on changing gut microbiota in order to prevent bacterial translocation and reduce ammonia scavengers have also been explored, especially various probiotics have been shown to reverse MHE and to prevent development of overt HE $(35,36)$. Probiotics have few side effects, but evidence supporting their efficacy on mortality and quality of life is still scarce as well as dose and duration of treatment required needs further investigation (37). L-ornithine, $\mathrm{L}$-aspartate is likewise beneficial in prevention of recurrent $\mathrm{HE}$, but at present insufficiently assessed for MHE (38). Clinical studies investigating the effects of antiinflammatory drugs in both MHE and HE in both the acute situation and as long-term prophylaxis are in demand, but NSAIDS remains contra indicated in cirrhotic patients with an increased risk of gastro intestinal bleeding. In the present study, we also looked for relations between abnormal PHES and clinical measures of disease severity. Apart from our finding that more patients with abnormal PHES had experienced a previous episode of HE, we found no evidence to support the assumption that an abnormal PHES score is related to a more advanced liver disease. MHE has not been shown to be related to an increased mortality, while overt HE predicts a poorer outcome for patients (39). However, MHE is associated with a decreased quality of life and a high burden of health care $(3,40)$. New knowledge into the impact of MHE on health care resources, and risk of hospital admission during long-term follow-up should be the focus in future research.

Data for the present study was uniformly and consecutively collected according to study protocol in a real-time setting. However, the cohort was highly selected due to eligibility criteria for a randomized trial of portal hypertension and bacterial translocation, and presence or absence of MHE was not part of in- or exclusion criteria. MHE is a dynamic entity, and assessment in clinical practice is challenged by various testing modalities, diagnostic heterogeneity and inter test variability. Absence of options for treatment, as well as lack of clinical application of guidelines for diagnosis in practice also challenge the day to day management of this patient group (6,41-44). Future clinical practice should focus on accurate testing and concise diagnosis as well as motivation to test available treatment options in a structured manner is in high need. Moreover, efforts should be made to prevent episodes of overt HE, and to improve the quality of life in MHE patients. Advancing self-support should also be a future focus point in research and clinic.

When exploring the pathogenesis of HE and MHE, long term studies with an epidemiological approach including patients from the time of diagnosis are preferable as this would provide new knowledge into aetiology and precipitating factors of $\mathrm{MHE}$ and $\mathrm{HE}$. A deeper insight into mechanisms facilitating and driving neurocognitive impairment in cirrhosis may allow for new advances in treatment and care.

\section{Acknowledgments}

The authors wish to thank Professor Marsha Morgan for invaluable academic discussions and feedback on scientific ideas and theories.

Funding: This trial was funded by The Research Foundation of The Capital Region of Denmark, Novo Nordisk Foundation (NNF13OC0006561), Aase \& Ejnar Danielsens Foundation, and Amager and Hvidovre Hospital Research Foundation.

\section{Footnote}

Provenance and Peer Review: This article was a free submission to the journal. The article has undergone external peer review.

Conflicts of Interest: All authors have completed the ICMJE uniform disclosure form (available at http://dx.doi. org/10.21037/tgh.2020.02.14). Dr. NK reports grants from Norgine Denmark A/S, during the conduct of the study; 
grants from Bridge Translational Excellence Program UCPH, outside the submitted work. Dr. FB reports grants from Ferring Pharmaceutical, outside the submitted work. The other authors have no conflicts of interest to declare.

Ethical Statement: The authors are accountable for all aspects of the work in ensuring that questions related to the accuracy or integrity of any part of the work are appropriately investigated and resolved. The study was conducted in accordance with the Declaration of Helsinki (as revised in 2013). The randomized clinical trial was approved by the Danish Medicines Agency (EudraCT No. 2012002890-71) and by the Scientific Ethics Committee of the Capital Region of Denmark (journal No. H-2012-078). All participants gave informed written consent to participation in the trial.

Open Access Statement: This is an Open Access article distributed in accordance with the Creative Commons Attribution-NonCommercial-NoDerivs 4.0 International License (CC BY-NC-ND 4.0), which permits the noncommercial replication and distribution of the article with the strict proviso that no changes or edits are made and the original work is properly cited (including links to both the formal publication through the relevant DOI and the license). See: https://creativecommons.org/licenses/by-nc-nd/4.0/.

\section{References}

1. Jepsen P, Ott P, Andersen PK, et al. Clinical course of alcoholic liver cirrhosis: a Danish population-based cohort study. Hepatology 2010;51:1675-82.

2. Vilstrup H, Amodio P, Bajaj J, et al. Hepatic encephalopathy in chronic liver disease: 2014 Practice Guideline by the American Association for the Study of Liver Diseases and the European Association for the Study of the Liver. Hepatology 2014;60:715-35.

3. Labenz C, Baron JS, Toenges G, et al. Prospective evaluation of the impact of covert hepatic encephalopathy on quality of life and sleep in cirrhotic patients. Aliment Pharmacol Ther 2018;48:313-21.

4. Singh J, Sharma BC, Puri V, et al. Sleep disturbances in patients of liver cirrhosis with minimal hepatic encephalopathy before and after lactulose therapy. Metab Brain Dis 2017;32:595-605.

5. Olesen SS, Jackson CD, Morgan MY. Tools and tactics for improving diagnosis of hepatic encephalopathy. J Hepatol
2017;66:1327-8.

6. Morgan MY, Amodio P, Cook NA, et al. Qualifying and quantifying minimal hepatic encephalopathy. Metab Brain Dis 2016;31:1217-29.

7. Randolph C, Hilsabeck R, Kato A, et al. Neuropsychological assessment of hepatic encephalopathy: ISHEN practice guidelines. Liver Int 2009;29:629-35.

8. Weissenborn K, Ennen JC, Schomerus H, et al. Neuropsychological characterization of hepatic encephalopathy. J Hepatol 2001;34:768-73.

9. Pantham G, Post A, Venkat D, et al. A New Look at Precipitants of Overt Hepatic Encephalopathy in Cirrhosis. Dig Dis Sci 2017;62:2166-73.

10. Butterworth RF, Giguere JF, Michaud J, et al. Ammonia: key factor in the pathogenesis of hepatic encephalopathy. Neurochem Pathol 1987;6:1-12.

11. Kang DJ, Betrapally NS, Ghosh SA, et al. Gut microbiota drive the development of neuroinflammatory response in cirrhosis in mice. Hepatology 2016;64:1232-48.

12. Albillos A, Lario M, Alvarez-Mon M. Cirrhosis-associated immune dysfunction: distinctive features and clinical relevance. J Hepatol 2014;61:1385-96.

13. Mangas-Losada A, Garcia-Garcia R, Urios A, et al. Minimal hepatic encephalopathy is associated with expansion and activation of $\mathrm{CD}(4+) \mathrm{CD} 28(-)$, Th22 and Tfh and B lymphocytes. Sci Rep 2017;7:6683.

14. Montagnese S, Biancardi A, Schiff S, et al. Different biochemical correlates for different neuropsychiatric abnormalities in patients with cirrhosis. Hepatology 2011;53:558-66.

15. Felipo V, Urios A, Montesinos E, et al. Contribution of hyperammonemia and inflammatory factors to cognitive impairment in minimal hepatic encephalopathy. Metab Brain Dis 2012;27:51-8.

16. Jain L, Sharma BC, Srivastava S, et al. Serum endotoxin, inflammatory mediators, and magnetic resonance spectroscopy before and after treatment in patients with minimal hepatic encephalopathy. J Gastroenterol Hepatol 2013;28:1187-93.

17. Wunsch E, Koziarska D, Milkiewicz M, et al. In patients with liver cirrhosis, proinflammatory interleukins correlate with health-related quality of life irrespective of minimal hepatic encephalopathy. Eur J Gastroenterol Hepatol 2013;25:1402-7.

18. Kimer N, Pedersen JS, Busk TM, et al. Rifaximin has no effect on hemodynamics in decompensated cirrhosis: A randomized, double-blind, placebo-controlled trial. 
Hepatology 2017;65:592-603.

19. Kimer N, Pedersen JS, Tavenier J, et al. Rifaximin has minor effects on bacterial composition, inflammation, and bacterial translocation in cirrhosis: A randomized trial. J Gastroenterol Hepatol 2018;33:307-14.

20. Systems RD. Product information. 2016. Available online: http://www.rndsystems.com/products. Accessed May 18 2016.

21. Duner KI. A new kinetic single-stage Limulus amoebocyte lysate method for the detection of endotoxin in water and plasma. J Biochem Biophys Methods 1993;26:131-42.

22. Mortensen C, Jensen JS, Hobolth L, et al. Association of markers of bacterial translocation with immune activation in decompensated cirrhosis. Eur J Gastroenterol Hepatol 2014;26:1360-6.

23. Riggio O, Amodio P, Farcomeni A, et al. A Model for Predicting Development of Overt Hepatic Encephalopathy in Patients With Cirrhosis. Clin Gastroenterol Hepatol 2015;13:1346-52.

24. Mani AR, Montagnese S, Jackson CD, et al. Decreased heart rate variability in patients with cirrhosis relates to the presence and degree of hepatic encephalopathy. Am J Physiol Gastrointest Liver Physiol 2009;296:G330-8.

25. Montoliu C, Piedrafita B, Serra MA, et al. IL-6 and IL18 in blood may discriminate cirrhotic patients with and without minimal hepatic encephalopathy. J Clin Gastroenterol 2009;43:272-9.

26. Shawcross DL, Davies NA, Williams R, et al. Systemic inflammatory response exacerbates the neuropsychological effects of induced hyperammonemia in cirrhosis. J Hepatol 2004;40:247-54.

27. Odeh M, Sabo E, Srugo I, et al. Serum levels of tumor necrosis factor-alpha correlate with severity of hepatic encephalopathy due to chronic liver failure. Liver Int 2004;24:110-6.

28. Odeh M, Sabo E, Srugo I, et al. Relationship between tumor necrosis factor-alpha and ammonia in patients with hepatic encephalopathy due to chronic liver failure. Ann Med 2005;37:603-12.

29. Sharma BC, Singh J, Srivastava S, et al. Randomized controlled trial comparing lactulose plus albumin versus lactulose alone for treatment of hepatic encephalopathy. J Gastroenterol Hepatol 2017;32:1234-9.

30. Cauli O, Rodrigo R, Piedrafita B, et al. Inflammation and hepatic encephalopathy: ibuprofen restores learning ability in rats with portacaval shunts. Hepatology 2007;46:514-9.

31. Rodrigo R, Cauli O, Gomez-Pinedo U, et al.
Hyperammonemia induces neuroinflammation that contributes to cognitive impairment in rats with hepatic encephalopathy. Gastroenterology 2010;139:675-84.

32. Kimer N, Krag A, Moller S, et al. Systematic review with meta-analysis: the effects of rifaximin in hepatic encephalopathy. Aliment Pharmacol Ther 2014;40:123-32.

33. Gluud LL, Vilstrup H, Morgan MY. Nonabsorbable disaccharides for hepatic encephalopathy: A systematic review and meta-analysis. Hepatology 2016;64:908-22.

34. Gluud LL, Dam G, Borre M, et al. Oral branched-chain amino acids have a beneficial effect on manifestations of hepatic encephalopathy in a systematic review with meta-analyses of randomized controlled trials. J Nutr 2013;143:1263-8.

35. Sharma K, Pant S, Misra S, et al. Effect of rifaximin, probiotics, and 1-ornithine 1-aspartate on minimal hepatic encephalopathy: a randomized controlled trial. Saudi J Gastroenterol 2014;20:225-32.

36. Cao Q, Yu CB, Yang SG, et al. Effect of probiotic treatment on cirrhotic patients with minimal hepatic encephalopathy: A meta-analysis. Hepatobiliary Pancreat Dis Int 2018;17:9-16.

37. Dalal R, McGee RG, Riordan SM, et al. Probiotics for people with hepatic encephalopathy. Cochrane Database Syst Rev 2017;2:CD008716.

38. Varakanahalli S, Sharma BC, Srivastava S, et al. Secondary prophylaxis of hepatic encephalopathy in cirrhosis of liver: a double-blind randomized controlled trial of L-ornithine L-aspartate versus placebo. Eur J Gastroenterol Hepatol 2018;30:951-8.

39. Thomsen KL, Macnaughtan J, Tritto G, et al. Clinical and Pathophysiological Characteristics of Cirrhotic Patients with Grade 1 and Minimal Hepatic Encephalopathy. PLoS One 2016;11:e0146076.

40. Ridola L, Cardinale V, Riggio O. The burden of minimal hepatic encephalopathy: from diagnosis to therapeutic strategies. Ann Gastroenterol 2018;31:151-64.

41. Bajaj JS, Cordoba J, Mullen KD, et al. Review article: the design of clinical trials in hepatic encephalopathy-an International Society for Hepatic Encephalopathy and Nitrogen Metabolism (ISHEN) consensus statement. Aliment Pharmacol Ther 2011;33:739-47.

42. American Association for the Study of Liver Diseases; European Association for the Study of the Liver. Hepatic encephalopathy in chronic liver disease: 2014 practice guideline by the European Association for the Study of the Liver and the American Association for the Study of Liver 
Diseases. J Hepatol 2014;61:642-59.

43. Campagna F, Montagnese S, Ridola L, et al. The animal naming test: An easy tool for the assessment of hepatic encephalopathy. Hepatology 2017;66:198-208.

doi: $10.21037 / \operatorname{tgh} .2020 .02 .14$

Cite this article as: Kimer N, Gluud LL, Pedersen JS, Tavenier J, Møller S, Bendtsen F. The Psychometric Hepatic Encephalopathy Syndrome score does not correlate with blood ammonia, endotoxins or markers of inflammation in patients with cirrhosis. Transl Gastroenterol Hepatol 2021;6:8
44. Montagnese S, Angeli P, Craxi A. Between reality and the guidelines: A survey on perception, diagnosis and management of hepatic encephalopathy in 201 Italian specialist centres. Dig Liver Dis 2017;49:828-30. 